\title{
Machine Learning Tools to Predict Hot Injection Syntheses Outcomes for II-VI and IV-VI Quantum Dots
}

\author{
Fábio Baum, ${ }^{\mathrm{a}^{*}}$ Tatiane Pretto, ${ }^{\mathrm{a}}$ Ariadne Köche, ${ }^{\mathrm{a}}$ Marcos José Leite Santos ${ }^{\mathrm{ab} *}$ \\ aPrograma de Pós-Graduação em Ciência dos Materiais, Universidade Federal do Rio Grande do \\ Sul (UFRGS); \\ bPrograma de Pós-Graduação em Química, Instituto de Química, Universidade Federal do Rio \\ Grande do Sul (UFRGS).
}

\section{Supplementary Information}

Table S 1. Variables used to feed the Machine Learning algorithms.

\begin{tabular}{|c|c|c|c|}
\hline Variable & Symbol & Unity & Brief definition \\
\hline Injection Temperature & $T_{\text {inj }}$ & ${ }^{\circ} \mathrm{C}$ & $\begin{array}{l}\text { Temperature of the solution in the } \\
\text { three-neck flask at the time of reaction. }\end{array}$ \\
\hline Growth Temperature & $T_{\text {grw }}$ & ${ }^{\circ} \mathrm{C}$ & $\begin{array}{l}\text { Temperature of the reaction after the } \\
\text { injection. }\end{array}$ \\
\hline Time of Reaction & $t$ & $\min$ & $\begin{array}{l}\text { Time elapsed between the injection } \\
\text { and the quench of the reaction. }\end{array}$ \\
\hline Metal Source & M & - & $\begin{array}{l}\text { Chemical precursor of the metal, } \\
\text { typically a salt. }\end{array}$ \\
\hline Metal Amount & $\mathrm{M}_{\mathrm{g}}$ & g & $\begin{array}{l}\text { Amount, in grams, of the metal source } \\
\text { used in the reaction. }\end{array}$ \\
\hline Metal Molar Amount & $\mathrm{M}_{\mathrm{mmol}}$ & $\mathrm{mmol}$ & $\begin{array}{l}\text { Amount, in mmol, of the metal source } \\
\text { used in the reaction. }\end{array}$ \\
\hline Metal Concentration & [M] & $\mathrm{mmol} / \mathrm{g}$ & $\begin{array}{l}\text { Concentration of the metal source in } \\
\text { the reaction, given by the Metal Molar } \\
\text { Amount divided by the Total Amount. }\end{array}$ \\
\hline Chalcogen Source & $\mathrm{C}$ & - & Chemical precursor of the chalcogen. \\
\hline Chalcogen Amount & $\mathrm{C}_{\mathrm{g}}$ & g & $\begin{array}{l}\text { Amount, in grams, of the chalcogen } \\
\text { source used in the reaction. }\end{array}$ \\
\hline $\begin{array}{l}\text { Chalcogen Molar } \\
\text { Amount }\end{array}$ & $\mathrm{C}_{\mathrm{mmol}}$ & $\mathrm{mmol}$ & $\begin{array}{l}\text { Amount, in mmol, of the chalcogen } \\
\text { source used in the reaction. }\end{array}$ \\
\hline $\begin{array}{l}\text { Chalcogen } \\
\text { Concentration }\end{array}$ & [C] & $\mathrm{mmol} / \mathrm{g}$ & $\begin{array}{l}\text { Concentration of the chalcogen source } \\
\text { in the reaction, given by the Chalcogen } \\
\text { Molar Amount divided by the Total } \\
\text { Amount. }\end{array}$ \\
\hline $\begin{array}{l}\text { Metal/Chalcogen } \\
\text { Ratio }\end{array}$ & $\mathrm{M} / \mathrm{C}$ & - & $\begin{array}{l}\text { Molar ratio between metal and } \\
\text { chalcogen sources, given by the Metal } \\
\text { Molar Amount divided by the } \\
\text { Chalcogen Molar Amount. }\end{array}$ \\
\hline Carboxylic Acid & CA & - & $\begin{array}{l}\text { Carboxylic Acid used in the reaction. In } \\
\text { the case of the absence of carboxylic } \\
\text { acid, a } 0 \text { is used in its place on the } \\
\text { database. }\end{array}$ \\
\hline
\end{tabular}




\begin{tabular}{|c|c|c|c|}
\hline $\begin{array}{l}\text { Carboxylic Acid } \\
\text { Amount }\end{array}$ & $\mathrm{CA}_{\mathrm{g}}$ & $g$ & $\begin{array}{l}\text { Amount, in grams, of the carboxylic } \\
\text { acid used in the reaction. }\end{array}$ \\
\hline $\begin{array}{l}\text { Carboxylic Acid Molar } \\
\text { Amount }\end{array}$ & $\mathrm{CA}_{\mathrm{mmol}}$ & $\mathrm{mmol}$ & $\begin{array}{l}\text { Amount, in mmol, of the carboxylic acid } \\
\text { used in the reaction. }\end{array}$ \\
\hline $\begin{array}{l}\text { Metal/Carboxylic Acid } \\
\text { Ratio }\end{array}$ & $\mathrm{M} / \mathrm{CA}$ & - & $\begin{array}{l}\text { Molar ratio between metal and } \\
\text { carboxylic acid, given by the Metal } \\
\text { Molar Amount divided by the } \\
\text { Carboxylic Acid Molar Amount. }\end{array}$ \\
\hline Amine & A & - & $\begin{array}{l}\text { Amine used in the reaction. In the case } \\
\text { of the absence of amines, a } 0 \text { is used } \\
\text { in its place on the database. }\end{array}$ \\
\hline Amine Amount & $A_{g}$ & g & $\begin{array}{l}\text { Amount, in grams, of the amine used in } \\
\text { the reaction. }\end{array}$ \\
\hline Phosphine & $P$ & - & $\begin{array}{l}\text { Phosphine used in the reaction. In the } \\
\text { case of the absence of phosphines, a } \\
0 \text { is used in its place on the database. }\end{array}$ \\
\hline Phosphine Amount & $P_{g}$ & g & $\begin{array}{l}\text { Amount, in grams, of the phosphine } \\
\text { used in the reaction. }\end{array}$ \\
\hline $\begin{array}{l}\text { Phosphine Molar } \\
\text { Amount }\end{array}$ & $P_{\mathrm{mmol}}$ & $\mathrm{mmol}$ & $\begin{array}{l}\text { Amount, in mmol, of the phosphine } \\
\text { used in the reaction. }\end{array}$ \\
\hline $\begin{array}{l}\text { Chalcogen/Phosphine } \\
\text { Ratio }\end{array}$ & $C / P$ & - & $\begin{array}{l}\text { Molar ratio between chalcogen and } \\
\text { phosphine, given by the Chalcogen } \\
\text { Molar Amount divided by the } \\
\text { Phosphine Molar Amount. }\end{array}$ \\
\hline Solvent 1 & $S^{1}$ & - & $\begin{array}{l}\text { Solvent used in the reaction. In the } \\
\text { case of the absence of solvents, a } 0 \text { is } \\
\text { used in its place on the database. }\end{array}$ \\
\hline Solvent 1 Amount & $S^{1}{ }_{g}$ & $g$ & $\begin{array}{l}\text { Amount, in grams, of the solvent used } \\
\text { in the reaction. }\end{array}$ \\
\hline Solvent 2 & $S^{2}$ & - & $\begin{array}{l}\text { Second solvent used in the reaction, } \\
\text { when solvent combination was used. } \\
\text { In the case of the absence of this } \\
\text { solvent, a } 0 \text { is used in its place on the } \\
\text { database. }\end{array}$ \\
\hline Solvent 2 Amount & $\mathrm{S}^{2}{ }_{\mathrm{g}}$ & g & $\begin{array}{l}\text { Amount, in grams, of the second } \\
\text { solvent used in the reaction. }\end{array}$ \\
\hline Total Amount & $\mathrm{T}$ & g & $\begin{array}{l}\text { The sum of all components of the } \\
\text { reaction, in grams. }\end{array}$ \\
\hline Diameter & D & $\mathrm{nm}$ & $\begin{array}{l}\text { The final diameter of the quantum dot } \\
\text { obtained in each reaction. }\end{array}$ \\
\hline
\end{tabular}


Table S 2. Description of the dataset and range of the variables.

\begin{tabular}{|c|c|c|c|c|c|}
\hline Feature & $\mathrm{CdS}$ & CdSe & $\mathrm{PbS}$ & PbSe & $\mathrm{ZnSe}$ \\
\hline Papers & 17 & 36 & 25 & 18 & 28 \\
\hline Syntheses & 161 & 357 & 114 & 84 & 121 \\
\hline $\mathrm{T}_{\mathrm{inj}}$ & $150-300$ & $20-330$ & $30-210$ & $30-220$ & $110-330$ \\
\hline$T_{\text {grw }}$ & $150-260$ & $45-330$ & $5-220$ & $30-220$ & $110-320$ \\
\hline $\mathrm{t}$ & $0,17-360$ & $0,003-4180$ & $0,08-60$ & $0,1-200$ & $0,17-900$ \\
\hline M & $\begin{array}{l}\text { cadmium stearate } \\
\text { cadmium nitrate } \\
\text { tetrahydrate } \\
\text { cadmium chloride } \\
\text { cadmium oxide }\end{array}$ & $\begin{array}{c}\text { cadmium acetate } \\
\text { cadmium acetate } \\
\text { dihydrate } \\
\text { cadmium oxide } \\
\text { cadmium stearate } \\
\text { dimethylcadmium }\end{array}$ & $\begin{array}{c}\text { lead acetate trihydrate } \\
\text { lead chloride } \\
\text { lead stearate } \\
\text { lead oxide }\end{array}$ & $\begin{array}{l}\text { lead acetate } \\
\text { trihydrate } \\
\text { lead chloride } \\
\text { lead oxide } \\
\text { lead stearate }\end{array}$ & $\begin{array}{c}\text { zinc oxide } \\
\text { zinc nonanoate } \\
\text { zinc acetate } \\
\text { heptahydrate } \\
\text { zinc stearate } \\
\text { zinc carbonate } \\
\text { diethylzinc } \\
\text { zinc chloride } \\
\text { zinc acetate }\end{array}$ \\
\hline$M_{g}$ & $0,0128-1,54$ & $0,01-1,9$ & $0,088-18$ & $0,077-2,5$ & $0,01-2,53$ \\
\hline $\mathrm{M}_{\mathrm{mmol}}$ & $0,1-11,99$ & $0,086-13,35$ & $0,3596-80,6452$ & $0,1-11,2$ & $0,016-7,03$ \\
\hline [M] & $0,004-1,146$ & $0,0066-1,72$ & $0,0143-1,569$ & $0,0161-0,3116$ & $0,0019-0,48$ \\
\hline C & $\begin{array}{c}\text { sulfur } \\
\text { thioacetamide } \\
\text { thiourea }\end{array}$ & selenium & $\begin{array}{c}\text { bis(trimethylsilyl)sulfide } \\
\text { diphenylthiourea } \\
\text { Sulfur } \\
\text { Thioacetamide }\end{array}$ & $\begin{array}{l}\text { bis(trimethylsilyl) } \\
\text { selenide } \\
\text { selenium }\end{array}$ & $\begin{array}{c}\text { cyclo-hexeno-1.2.3- } \\
\text { selenadiazole } \\
\text { Se-octylamine- } \\
\text { oleylamine complex } \\
\text { selenium } \\
\text { selenium dioxide }\end{array}$ \\
\hline $\mathrm{C}_{\mathrm{g}}$ & $0,0016-0,25$ & $0,00395-0,79$ & $0,006-7,1$ & $0,008-1,5$ & $0,0016-1,0$ \\
\hline $\mathrm{C}_{\mathrm{mmol}}$ & $0,05-7,813$ & $0,05-9,99$ & $0,056-39,78$ & $0,1-19$ & $0,02-12,66$ \\
\hline$[\mathrm{C}]$ & $0,004-0,479$ & $0,0044-12,4$ & $0,0082-0,5756$ & $0,0161-0,67$ & $0,002-1,04$ \\
\hline $\mathrm{M} / \mathrm{C}$ & $0,2-20$ & $0,1-6,0$ & $0,98-22,12$ & $0,125-7,91$ & $0,77-32,3$ \\
\hline CA & $\begin{array}{l}\text { no ligand } \\
\text { lauric acid } \\
\text { oleic acid } \\
\text { stearic acid }\end{array}$ & $\begin{array}{l}\text { no ligand } \\
\text { oleic acid } \\
\text { stearic acid } \\
\text { benzoic acid } \\
\text { myristic acid }\end{array}$ & $\begin{array}{l}\text { no ligand } \\
\text { behenic acid } \\
\text { erucic acid } \\
\text { oleic acid }\end{array}$ & $\begin{array}{l}\text { no ligand } \\
\text { ascorbic acid } \\
\text { oleic acid }\end{array}$ & $\begin{array}{c}\text { no ligand } \\
\text { decanoic acid } \\
\text { nonanoic acid } \\
\text { octadecylphosphonic } \\
\text { acid } \\
\text { oleic acid }\end{array}$ \\
\hline $\mathrm{CA}_{\mathrm{g}}$ & $0-18,869$ & $0-16,11$ & $0-62$ & $0-7,5$ & $0-15,1$ \\
\hline $\mathrm{CA}_{\mathrm{mmol}}$ & $0-24$ & $0-57,03$ & $0-219,5$ & $0-26,55$ & $0,69-53,5$ \\
\hline $\mathrm{M} / \mathrm{CA}$ & $0-0,43$ & $0-3,33$ & $0,01-3,95$ & $0-6,32$ & $0,086-0,50$ \\
\hline$A$ & $\begin{array}{c}\text { no amine } \\
\text { dodecylamine } \\
\text { oleylamine } \\
\text { triethanolamine }\end{array}$ & $\begin{array}{c}\text { no amine } \\
\text { hexadecylamine } \\
\text { dodecylamine } \\
\text { dioctylamine } \\
\text { oleylamine } \\
\text { octadecylamine } \\
\end{array}$ & $\begin{array}{l}\text { no amine } \\
\text { oleylamine }\end{array}$ & $\begin{array}{l}\text { no amine } \\
\text { oleylamine }\end{array}$ & $\begin{array}{c}\text { no amine } \\
\text { decylamine } \\
\text { hexadecylamine } \\
\text { octadecylamine } \\
\text { oleylamine }\end{array}$ \\
\hline$A_{g}$ & $0-27,4$ & $0-10$ & $0-22,8$ & $0-12,51$ & $0-10,16$ \\
\hline$P$ & $\begin{array}{l}\text { no phosphine } \\
\text { trioctylphosphine }\end{array}$ & $\begin{array}{l}\text { no phosphine } \\
\text { diphenylphosphine } \\
\text { tributylphosphine } \\
\text { trioctylphosphine } \\
\text { triphenylphosphine }\end{array}$ & $\begin{array}{l}\text { no phosphine } \\
\text { trioctylphosphine }\end{array}$ & $\begin{array}{l}\text { no phosphine } \\
\text { tributylphosphine } \\
\text { trioctylphosphine }\end{array}$ & $\begin{array}{l}\text { no phosphine } \\
\text { trioctylphosphine }\end{array}$ \\
\hline $\mathrm{P}_{\mathrm{g}}$ & $0-0,831$ & $0-20,78$ & $0-1,5$ & $0-16,62$ & $0-9,0$ \\
\hline $\mathrm{P}_{\mathrm{mmol}}$ & $0-2,242$ & $0-56,1$ & $0-4,05$ & $0-44,85$ & $2,42-24,3$ \\
\hline $\mathrm{C} / \mathrm{P}$ & $0,04-6,97$ & $0-4,264$ & 0,2215 & $0-8,95$ & $0,007-0,34$ \\
\hline $\mathrm{S}^{1}$ & $\begin{array}{c}\text { no solvent } 1 \\
\text { diesel } \\
\text { ethyleneglycol } \\
\text { n- } \\
\text { oleoylmorpholine } \\
\text { octadecene } \\
\text { paraffin oil } \\
\text { succinic acid } \\
\end{array}$ & $\begin{array}{c}\text { no solvent } 1 \\
\text { liquid paraffin } \\
\text { octadecene } \\
\text { pheynl ether } \\
\text { trioctylphosphine } \\
\text { oxide }\end{array}$ & $\begin{array}{l}\text { no solvent } 1 \\
\text { octadecene }\end{array}$ & $\begin{array}{c}\text { no solvent } 1 \\
\text { diphenyl ether } \\
\text { hexadecane } \\
\text { octadecene } \\
\text { trioctylphosphine } \\
\text { oxide }\end{array}$ & $\begin{array}{l}\text { no solvent } 1 \\
\text { diphenyl ether } \\
\text { octadecene } \\
\text { paraffin oil }\end{array}$ \\
\hline $\mathrm{S}_{\mathrm{g}}^{1}$ & $0-66,6$ & $0-101,4$ & $0-891$ & $0-23,7$ & $0-79,0$ \\
\hline$S^{2}$ & $\begin{array}{c}\text { no solvent } 2 \\
\text { 1-tetradecanol } \\
n- \\
\text { oleoylmorpholine } \\
\text { polyvinylpyrrolidon } \\
\mathrm{e} \\
\end{array}$ & $\begin{array}{c}\text { no solvent } 2 \\
\text { phosphinic acid } \\
\text { tributylphosphine } \\
\text { oxide } \\
\text { trioctylphosphine } \\
\text { oxide } \\
\end{array}$ & no solvent 2 & no solvent 2 & $\begin{array}{c}\text { no solvent } 2 \\
\text { paraffin oil }\end{array}$ \\
\hline$S_{g}^{2}$ & $0-5,36$ & $0-28,31$ & 0 & 0 & $0-23,75$ \\
\hline $\mathrm{T}$ & $4-682,84$ & $2,48-108,05$ & $1,93-69,1$ & $5,2-46,6$ & $2,65-104,0$ \\
\hline $\mathrm{D}$ & $1,23-12$ & $0,85-11,5$ & $1,57-80$ & $1,1-116,2$ & $2,0-9,6$ \\
\hline
\end{tabular}


Table S 3. Description of the sample diameter obtained from TEM or UV-Vis Spectra.

\begin{tabular}{|c|c|c|c|c|c|c|}
\hline Compound & \multicolumn{3}{|c|}{ UV-Vis } & \multicolumn{3}{c|}{ TEM } \\
\hline & Samples & $\begin{array}{c}\text { Mean/Std. } \\
\text { deviation }\end{array}$ & Range & Samples & $\begin{array}{c}\text { Mean/Std. } \\
\text { deviation }\end{array}$ & Range \\
\hline $\mathrm{CdS}$ & 82 & $4.75 / 1.20$ & $1.40-10.6$ & 79 & $4.05 / 1.51$ & $1.23-12.0$ \\
\hline $\mathrm{CdSe}$ & 100 & $2.14 / 0.69$ & $1.13-4.09$ & 257 & $3.41 / 1.76$ & $0.85-11.5$ \\
\hline $\mathrm{PbS}$ & 24 & $3.70 / 1.41$ & $1.57-6.17$ & 90 & $5.92 / 9.29$ & $1.95-80$ \\
\hline $\mathrm{PbSe}$ & 0 & - & - & 84 & $10.64 / 16.5$ & $1.1-116.2$ \\
\hline $\mathrm{ZnSe}$ & 24 & $3.96 / 0.56$ & $2.56-4.87$ & 97 & $4.09 / 1.43$ & $2.0-9.6$ \\
\hline
\end{tabular}
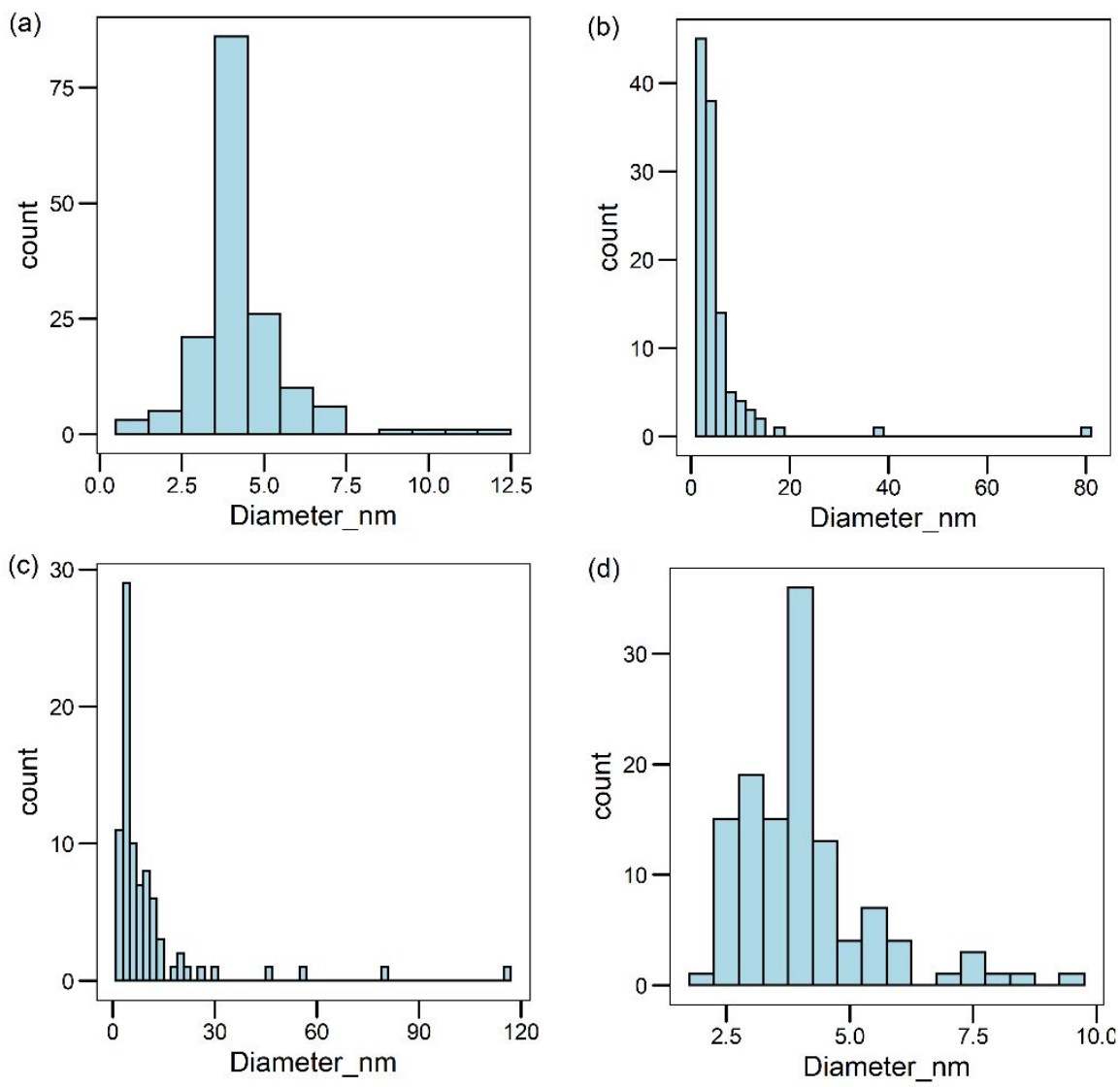

Figure S 1. Histograms of diameters of CdS (a), PbS (b), PbSe (c), and $\mathrm{ZnSe}(\mathrm{d})$ quantum dots found on literature and included on our database. 

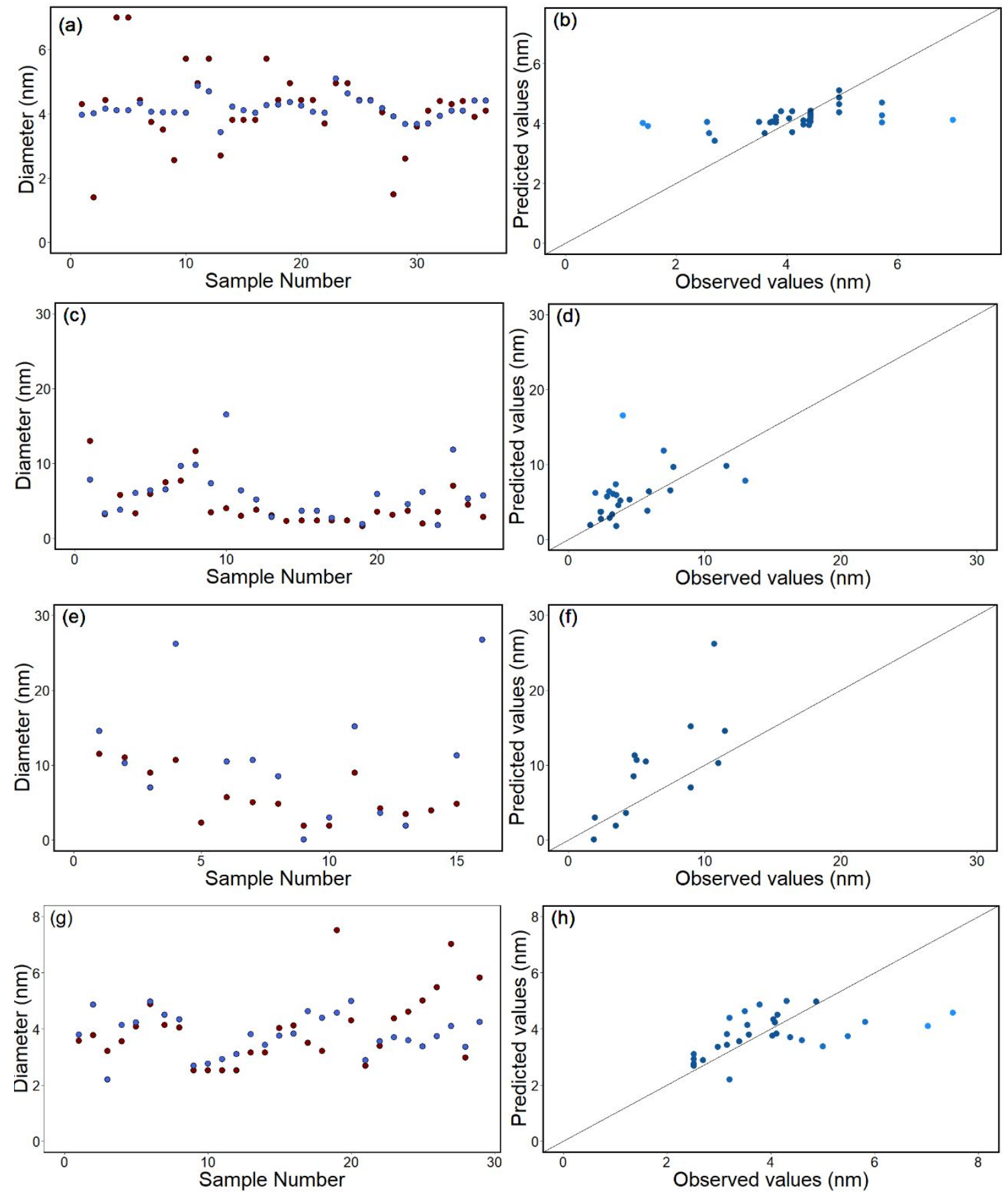

Figure S 2. Predicted (blue) vs observed (wine) plot of the multilinear regression model without interactions between variables for $\mathrm{CdS}$ ( $\mathrm{a}$ and $\mathrm{b}$ ), $\mathrm{PbS}$ (c and d), $\mathrm{PbSe}$ (e and f), and $\mathrm{ZnSe}(\mathrm{g}$ and $\mathrm{h})$ quantum dots. 

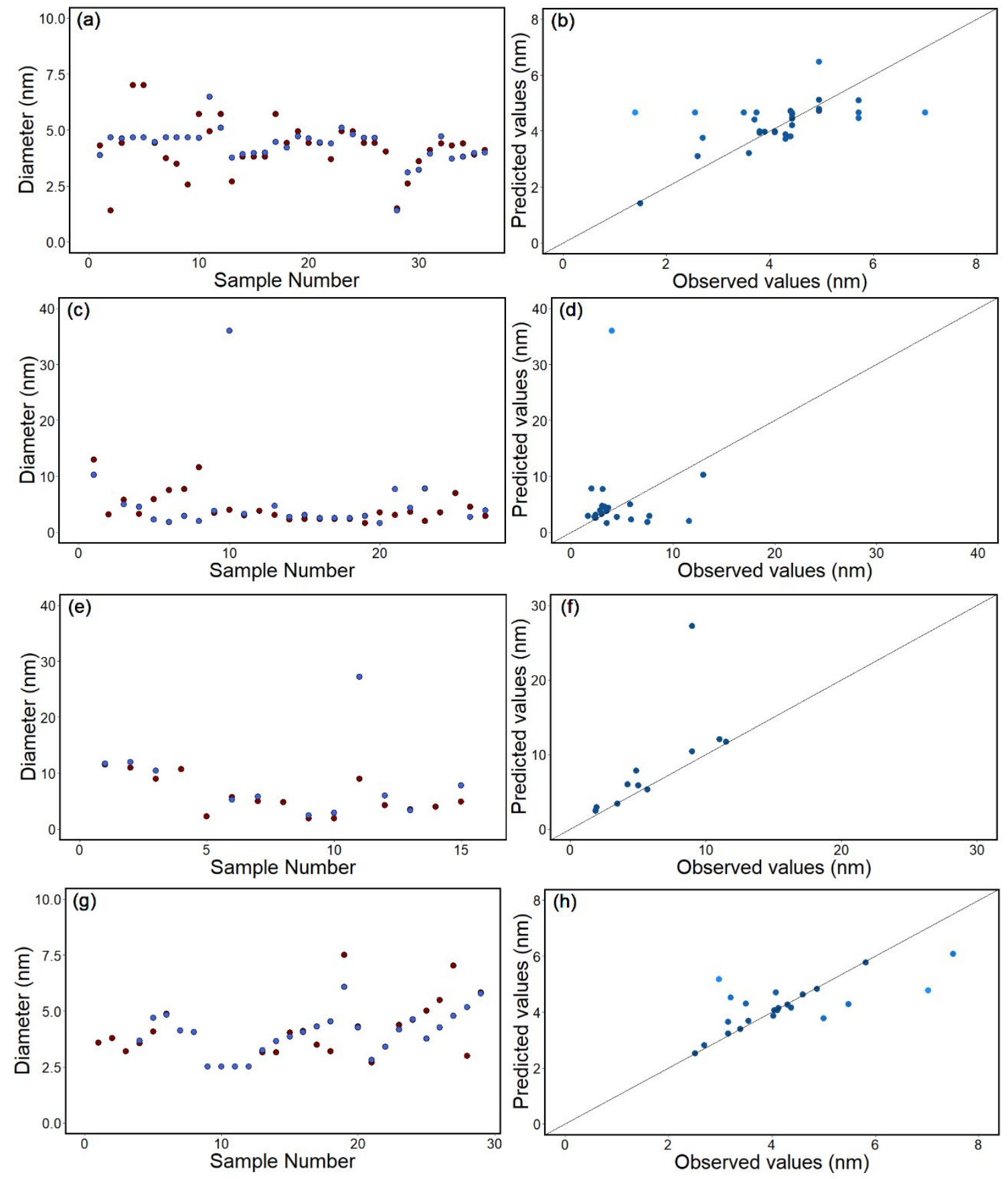

Figure S 3. Predicted (blue) vs observed (wine) plot of the multilinear regression model with interaction between variables $\mathrm{CdS}$ ( $\mathrm{a}$ and $\mathrm{b}$ ), $\mathrm{PbS}$ (c and d), $\mathrm{PbSe}$ (e and $\mathrm{f}$ ), and $\mathrm{ZnSe}$ ( $\mathrm{g}$ and $\mathrm{h}$ ) quantum dots. 

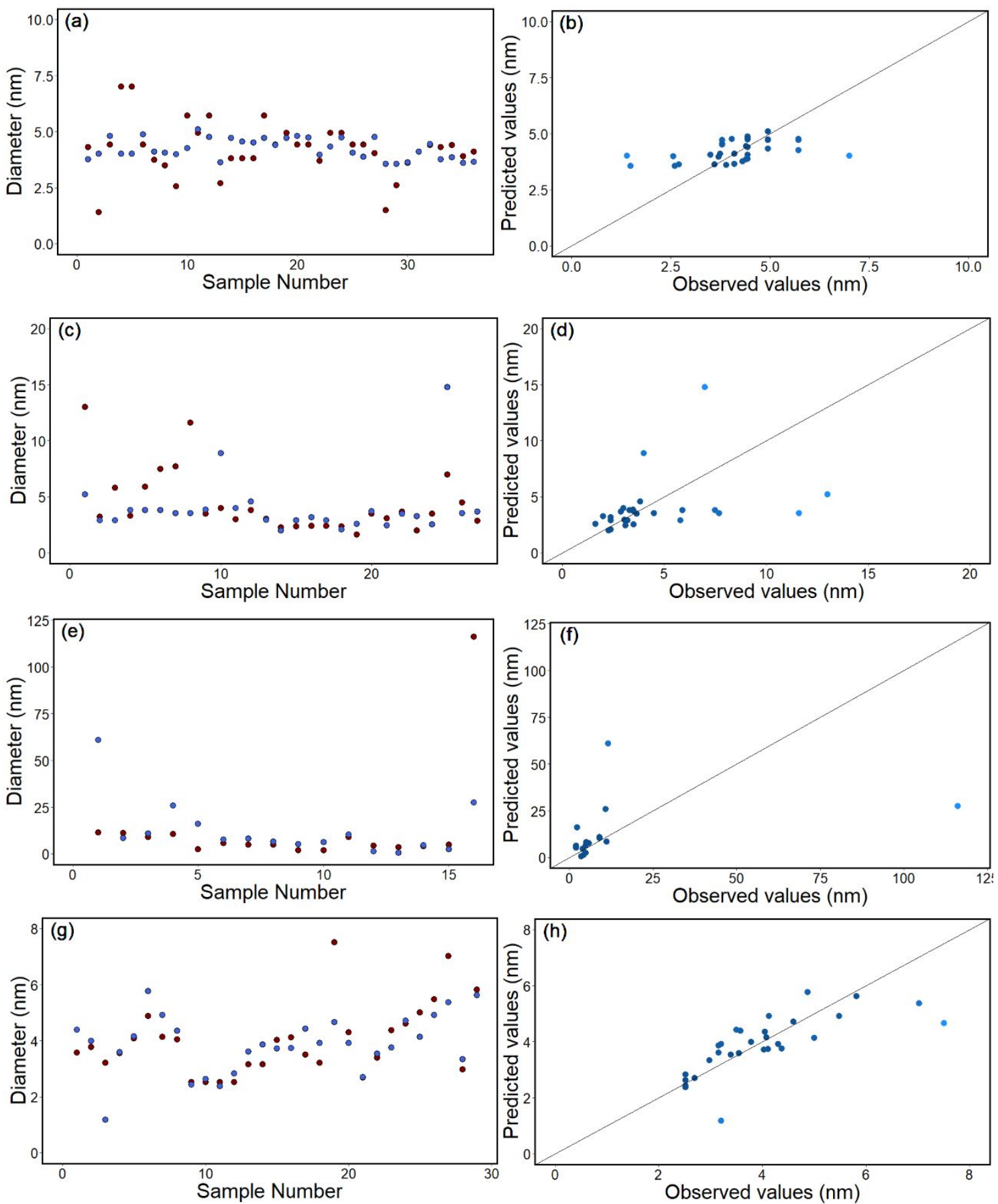

Figure S 4. Predicted vs observed plot of the smoothing splines regression model CdS (a and b), $\mathrm{PbS}$ (c and d), PbSe (e and f), and $\mathrm{ZnSe}$ (g and h) quantum dots. 


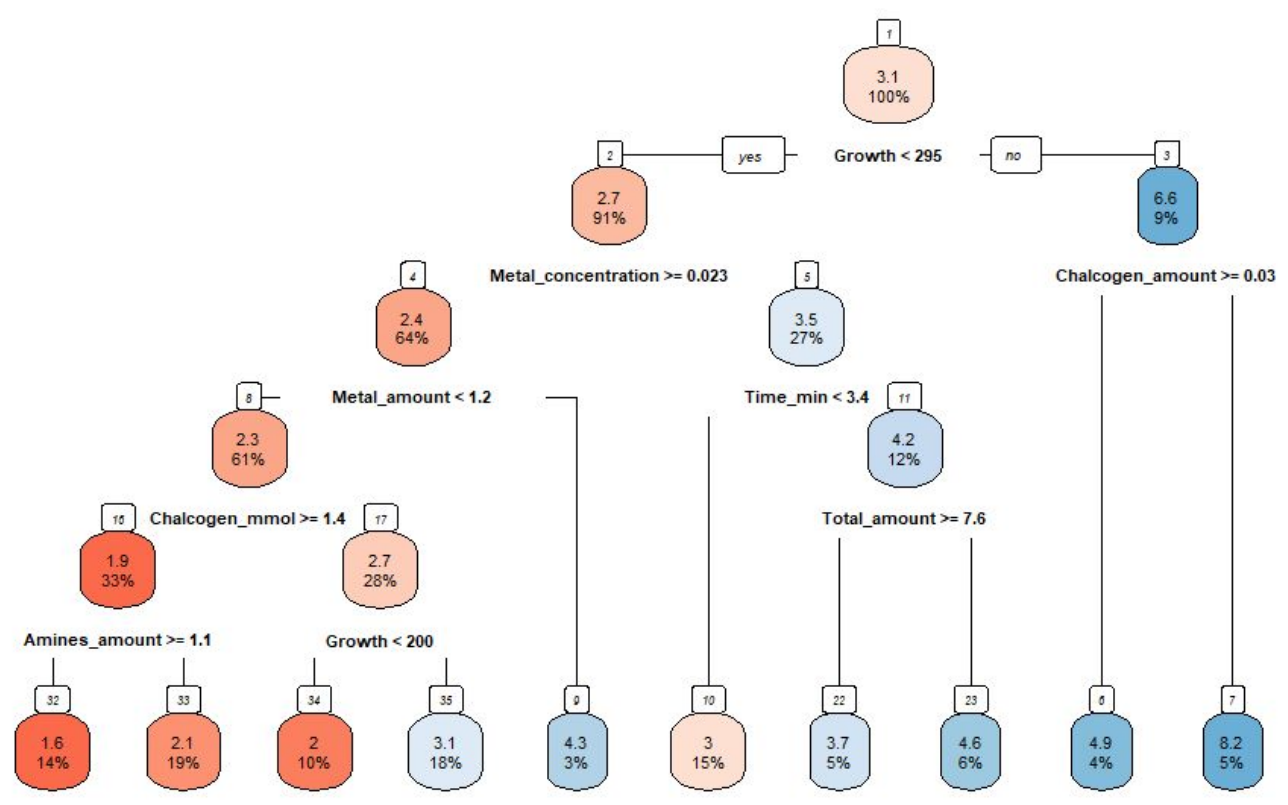

Figure S 5. Regression decision tree for quantum dots diameter as a function of all variables, either qualitative or quantitative. 

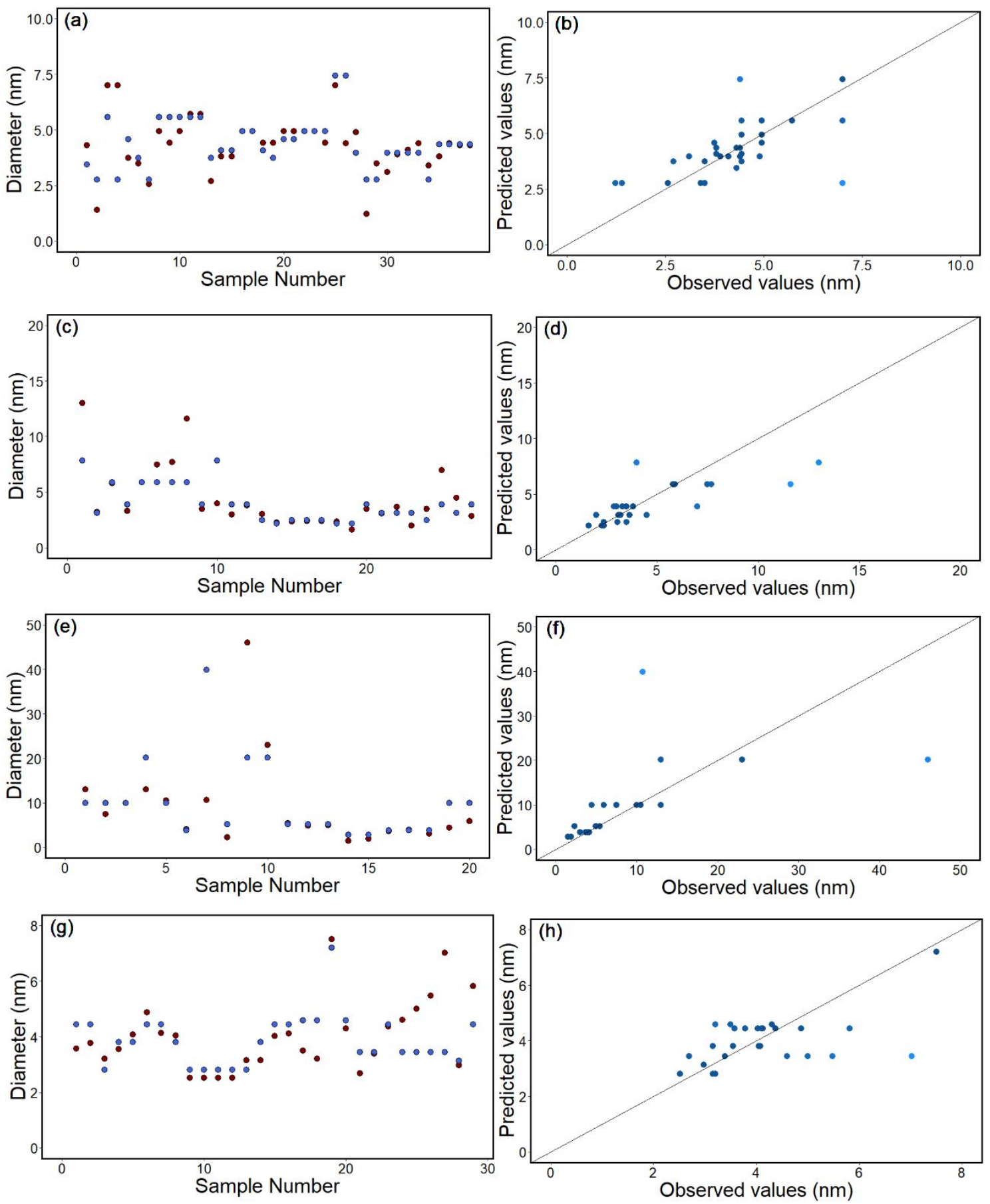

Figure S 6. Predicted (blue) vs observed (wine) plot of the decision tree regression model $\mathrm{CdS}(\mathrm{a}$ and $\mathrm{b}), \mathrm{PbS}$ (c and d), PbSe (e and f), and $\mathrm{ZnSe}(\mathrm{g}$ and $\mathrm{h}$ ) quantum dots. 

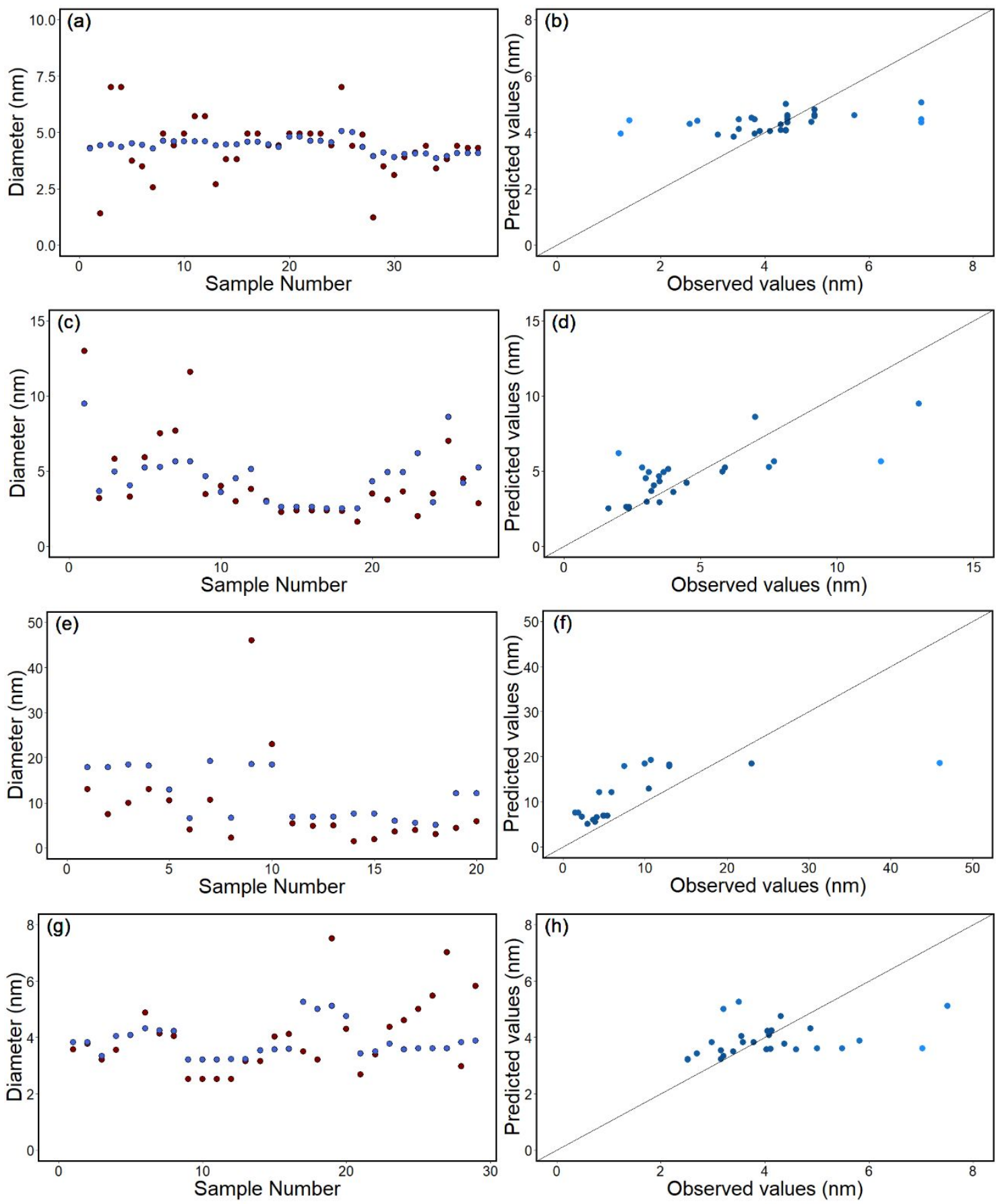

Figure S 7. Predicted (blue) vs observed (wine) plot of the random forest regression model CdS (a and b), PbS (c and d), PbSe (e and f), and $\mathrm{ZnSe}$ (g and h) quantum dots. 

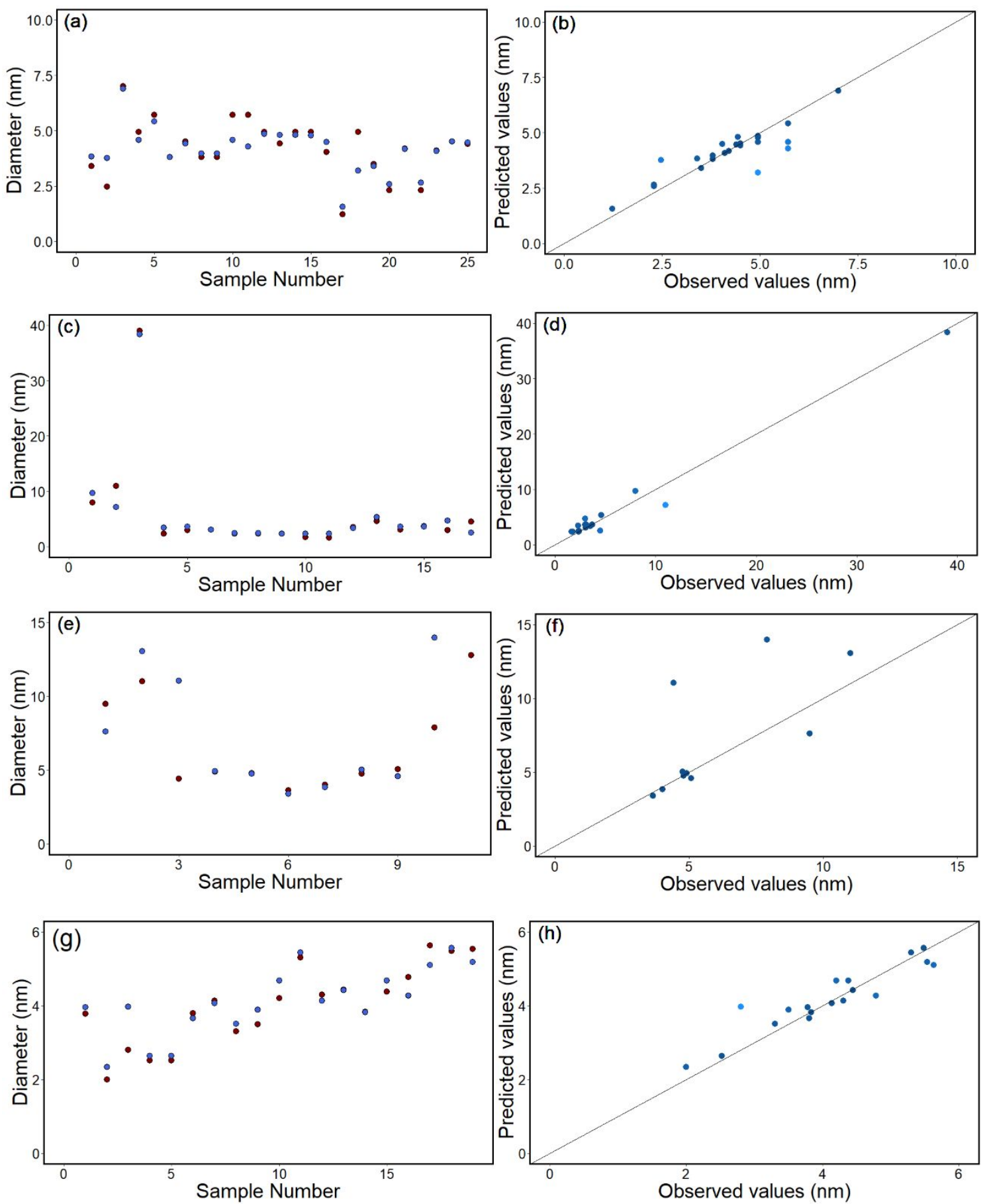

Figure S 8. Predicted vs observed plot of the gradient boosting machine regression model for $\mathrm{CdS}$ ( $\mathrm{a}$ and $\mathrm{b}$ ), $\mathrm{PbS}$ (c and d), PbSe (e and f), and $\mathrm{ZnSe}$ (g and h) quantum dots. 

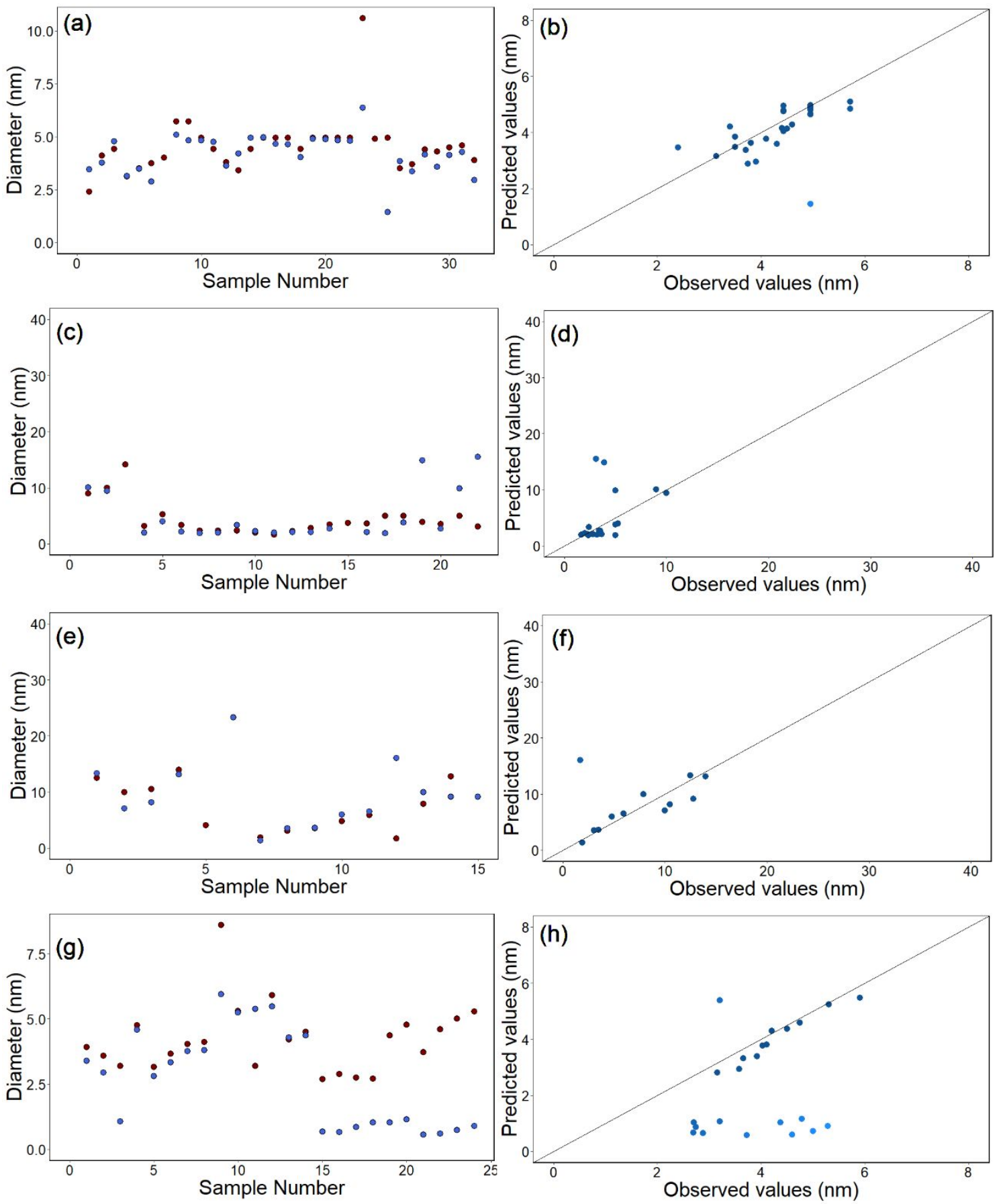

Figure S 9. Deep learning regression models for $\mathrm{CdS}$ (a and b), $\mathrm{PbS}$ (e and $\mathrm{f}), \mathrm{PbSe}(\mathrm{g}$ and $\mathrm{h}$ ), and $\mathrm{ZnSe}$ (i and j) quantum dots. 
Table S 4. Performance parameters of each algorithm for each compound.

\begin{tabular}{|c|c|c|c|c|c|c|c|}
\hline Compound & Model & \multicolumn{3}{|c|}{ Train data } & \multicolumn{3}{|c|}{ Test data } \\
\hline & & $\mathrm{R}^{2}$ & RMSE & MAE & $\mathrm{R}^{2}$ & RMSE & MAE \\
\hline $\mathrm{CdS}$ & Multilinear N. I. & 0.1711 & 0.8791 & 0.6303 & 0.0268 & 0.9715 & 0.6497 \\
\hline $\mathrm{CdS}$ & Multilinear W. I. & 0.5624 & 0.6711 & 0.4173 & 0.0020 & 235.164 & 63.435 \\
\hline $\mathrm{CdS}$ & $\begin{array}{c}\text { Smoothing } \\
\text { Splines }\end{array}$ & 0.1702 & 1.2734 & 0.7476 & & & \\
\hline $\mathrm{CdS}$ & Decision Tree & 0.5506 & 0.9996 & 0.6810 & 0.2141 & 1.2832 & 0.7471 \\
\hline $\mathrm{CdS}$ & Random Forest & 0.8497 & 0.6038 & 0.3755 & 0.3949 & 0.9799 & 0.5478 \\
\hline $\mathrm{CdS}$ & GBM & 0.9887 & 0.1510 & 0.0963 & 0.7442 & 0.6277 & 0.4111 \\
\hline $\mathrm{CdS}$ & Deep Learning & 0.8844 & 0.6112 & 0.3958 & 0.7286 & 0.4730 & 0.3683 \\
\hline $\mathrm{CdSe}$ & Multilinear N. I. & 0.3891 & 1.3462 & 0.9618 & 0.2430 & 2.6505 & 1.276 \\
\hline $\mathrm{CdSe}$ & Multilinear W. I. & 0.9669 & 0.2129 & 0.1437 & 0.0050 & 63204.5 & 10523.9 \\
\hline $\mathrm{CdSe}$ & $\begin{array}{l}\text { Smoothing } \\
\text { Splines }\end{array}$ & 0.5845 & 1.5034 & 1.0507 & & & \\
\hline $\mathrm{CdSe}$ & Decision Tree & 0.7289 & 0.8706 & 0.6034 & 0.4688 & 1.3263 & 0.8355 \\
\hline $\mathrm{CdSe}$ & Random Forest & 0.9429 & 0.3571 & 0.1844 & 0.8485 & 1.0420 & 0.5397 \\
\hline $\mathrm{CdSe}$ & GBM & 0.9972 & 0.0892 & 0.0509 & 0.9335 & 0.4961 & 0.2550 \\
\hline $\mathrm{CdSe}$ & Deep Learning & 0.7763 & 1.2192 & 0.5607 & 0.8304 & 1.0084 & 0.5961 \\
\hline $\mathrm{PbS}$ & Multilinear N. I. & 0.4643 & 1.5734 & 1.204 & 0.2854 & 11.9612 & 3.8104 \\
\hline $\mathrm{PbS}$ & Multilinear W. I. & 0.9978 & 2.779 & 2.028 & 0.0051 & 16200.7 & 3014.8 \\
\hline $\mathrm{PbS}$ & $\begin{array}{c}\text { Smoothing } \\
\text { Splines }\end{array}$ & 0.3130 & 6.9113 & 2.5204 & & & \\
\hline $\mathrm{PbS}$ & Decision Tree & 0.6734 & 9.1986 & 2.9342 & 0.2659 & 9.1986 & 2.9342 \\
\hline $\mathrm{PbS}$ & Random Forest & 0.9408 & 2.3442 & 0.9684 & 0.3912 & 7.3910 & 1.9189 \\
\hline $\mathrm{PbS}$ & GBM & 0.9811 & 1.1315 & 0.4040 & 0.9915 & 0.7919 & 0.5794 \\
\hline $\mathrm{PbS}$ & Deep Learning & 0.6521 & 5.1758 & 1.9315 & 0.9490 & 2.1156 & 1.2246 \\
\hline $\mathrm{PbSe}$ & Multilinear N. I. & 0.2921 & 12.5460 & 8.6247 & 0.1516 & 77.3998 & 37.7304 \\
\hline $\mathrm{PbSe}$ & Multilinear W. I. & 0.9998 & 24.757 & 9.535 & 0.1385 & 943724 & 430001 \\
\hline $\mathrm{PbSe}$ & $\begin{array}{c}\text { Smoothing } \\
\text { Splines }\end{array}$ & 0.3168 & 14.240 & 7.8967 & & & \\
\hline
\end{tabular}




\begin{tabular}{|c|c|c|c|c|c|c|c|}
\hline $\mathrm{PbSe}$ & Decision Tree & 0.4348 & 10.962 & 5.0320 & 0.4722 & 14.437 & 4.3347 \\
\hline $\mathrm{PbSe}$ & Random Forest & 0.8620 & 7.4507 & 3.3340 & 0.7590 & 11.8359 & 3.5942 \\
\hline $\mathrm{PbSe}$ & GBM & 0.0161 & 16.798 & 9.2082 & -2.682 & 5.5936 & 5.1701 \\
\hline $\mathrm{PbSe}$ & Deep Learning & 0.1974 & 12.042 & 4.7862 & 0.2817 & 16.403 & 4.1657 \\
\hline $\mathrm{ZnSe}$ & Multilinear N. I. & 0.3341 & 1.1571 & 0.8094 & 0.2208 & 0.9765 & 0.6915 \\
\hline $\mathrm{ZnSe}$ & Multilinear W. I. & 0.9967 & 2.5487 & 1.6975 & 0.0231 & 702.38 & 98.476 \\
\hline $\mathrm{ZnSe}$ & $\begin{array}{c}\text { Smoothing } \\
\text { Splines }\end{array}$ & 0.1350 & 1.2127 & 0.8126 & & & \\
\hline $\mathrm{ZnSe}$ & Decision Tree & 0.6222 & 1.0790 & 0.7537 & 0.3961 & 0.9677 & 0.7499 \\
\hline $\mathrm{ZnSe}$ & Random Forest & 0.9354 & 0.4406 & 0.2943 & 0.6597 & 0.6866 & 0.4593 \\
\hline $\mathrm{ZnSe}$ & GBM & 0.9990 & 0.3530 & 0.2803 & 0.8061 & 0.0954 & 0.0709 \\
\hline $\mathrm{ZnSe}$ & Deep Learning & 0.1974 & 1.3913 & 0.8735 & 0.1112 & 1.8493 & 1.2395 \\
\hline
\end{tabular}

Table S 5. DOIs used to compound the databases.

\begin{tabular}{|c|c|}
\hline \multicolumn{2}{|c|}{ CdS Database DOIs } \\
\hline 01. $10.1021 /$ acs.jpcc.6b01654 & 02. $1021 /$ acsnano.5b01859 \\
\hline 03. $10.1021 /$ ja204538f & 04. 1021/jp066392z \\
\hline 05. 10.1002/anie.200790059 & 06. $10.1007 / \mathrm{s} 10854-017-6731-\mathrm{y}$ \\
\hline 07. $10.1007 / \mathrm{s} 11671-006-9001-0$ & 08. 10.1021/acs.chemmater.9b04009 \\
\hline 09. $10.1021 / 1 \mathrm{a} 8005986$ & 10. $10.1016 /$ j.matchar.2006.05.010 \\
\hline 11. $10.1021 / \mathrm{ja} 0357902$ & 12. $10.1016 /$ j.mssp.2013.06.007 \\
\hline 13. 10.1016/j.optlastec.2019.03.001 & 14. 10.1016/j.colsurfa.2006.04.019 \\
\hline 15. $10.1016 /$ j.jcis.2009.10.030 & 16. $10.1166 /$ jnn.2010.2642 \\
\hline 17. $10.1016 /$ j.matlet.2011.01.077 & \\
\hline \multicolumn{2}{|c|}{ CdSe Database DOIs } \\
\hline 01. 10.1021/jp3092013 & 02. $10.1016 /$ j.colsurfa.2016.01.002 \\
\hline 03. $10.1021 /$ acs.jpclett. $7 \mathrm{~b} 01238$ & 04. $10.1016 /$ j.colsurfa.2014.07.045 \\
\hline 05. $10.1016 /$ j.cej.2017.02.154 & 06. $10.1021 /$ jp2035955 \\
\hline 07. $10.1155 / 2012 / 952610$ & 08. $10.1021 / \mathrm{cm} 900248 \mathrm{~b}$ \\
\hline 09. $10.1038 /$ srep24177 & 10. $10.1021 /$ ja00072a025 \\
\hline 11. $10.1021 / j p 063126 \mathrm{c}$ & 12. $10.1021 / \mathrm{nl} 0155126$ \\
\hline 13. $10.1021 / \mathrm{jp} 027160 \mathrm{c}$ & 14. $10.1246 / \mathrm{cl} .2005 .1004$ \\
\hline 15. $10.1016 /$ j.optmat.2017.01.041 & 16. $10.1021 / \mathrm{n} 10496724$ \\
\hline 17. $10.1039 / \mathrm{c} 4 \mathrm{ta} 00285 \mathrm{~g}$ & 18. $10.1016 /$ j.colsurfa.2005.07.017 \\
\hline 19. $10.1021 / j p 0542890$ & 20. $10.1021 /$ ja300132p \\
\hline 21. $10.1021 /$ acs chemmater. 5 b04790 & 22. $10.1021 / \mathrm{jp} 803746 b$ \\
\hline 23. $10.1063 / 1.3117221$ & 24. $10.1021 / \mathrm{ja} 017002 j$ \\
\hline
\end{tabular}




\begin{tabular}{|c|c|}
\hline 25. $10.1166 /$ jnn.2010.2564 & 26. $10.1021 /$ jp $809360 \mathrm{n}$ \\
\hline 27. 10.1016/j.colsurfa.2007.08.014 & 28. $10.1088 / 0957-4484 / 18 / 40 / 405603$ \\
\hline 29. $10.1088 / 2053-1591 / 3 / 7 / 075904$ & 30. $10.1021 / \mathrm{nl} 035211 \mathrm{r}$ \\
\hline 31. $10.1021 / j p 2086818$ & 32. $10.1021 /$ ja0458219 \\
\hline 33. $10.1021 / \mathrm{cm} 2008686$ & 34. 10.1016/j.jlumin.2006.04.009 \\
\hline \multicolumn{2}{|c|}{ PbS Database DOIs } \\
\hline 01. $10.1021 / \mathrm{ja} 0357902$ & 02. $10.1002 /$ adma.200305395 \\
\hline 03. $10.1016 /$ j.matlet.2006.01.007 & 04. $10.1088 / 0957-4484 / 19 / 34 / 345602$ \\
\hline 05. $10.1039 / \mathrm{B} 917833 \mathrm{C}$ & 06. 10.1021/acs.jpclett.5b00689 \\
\hline 07. $10.1021 /$ acsenergylett.6b00294 & 08. $10.1039 /$ C5TA08668J \\
\hline 09. $10.1166 /$ sam.2016.2513 & 10. $10.1039 / \mathrm{C} 6 \mathrm{CP} 02119 \mathrm{~K}$ \\
\hline 11. $10.1039 /$ C6RA20165B & 12. $10.1038 /$ srep24908. \\
\hline 13. $10.1039 / \mathrm{C} 7 \mathrm{NR} 03698 \mathrm{~A}$ & 14. $10.1002 / \mathrm{ppsc} .201600242$ \\
\hline 15. $10.1021 /$ acs.chemmater.6b04789 & 16. $10.1002 / \mathrm{smll} .201602956$ \\
\hline 17. $10.1039 / \mathrm{C} 6 \mathrm{TC} 05329 \mathrm{G}$ & 18. $10.1039 / \mathrm{C} 8 \mathrm{CP} 00767 \mathrm{E}$ \\
\hline 19. $10.3390 / \mathrm{ma} 12244221$ & 20. $10.1103 /$ physrevb.100.235411 \\
\hline 21. $10.1016 /$ j.jiec.2019.03.019 & 22. $10.1002 /$ slct.201904141 \\
\hline 23. $10.1039 / \mathrm{C} 9 \mathrm{NR} 07101 \mathrm{~F}$ & 24. 10.1016/j.solmat.2019.110363 \\
\hline 25. $10.1039 / \mathrm{C} 5 \mathrm{CC} 06955 \mathrm{~F}$ & 26. \\
\hline \multicolumn{2}{|c|}{ PbSe Database DOIs } \\
\hline 01. $10.1002 /$ asia.201201154 & 02. $10.1007 / \mathrm{s} 10854-016-5080-6$ \\
\hline 03. 10.1016/j.matpr.2015.04.043 & 04. $10.1021 / \mathrm{cm} 049476 \mathrm{y}$ \\
\hline 05. $10.1021 / \mathrm{cm} 202796 \mathrm{~s}$ & 06. $10.1021 / \mathrm{cm} 401083 \mathrm{~g}$ \\
\hline 07. $10.1021 / \mathrm{ja} 061644 \mathrm{v}$ & 08. $10.1021 /$ jacs.5b10273 \\
\hline 09. $10.1021 / \mathrm{jp} 102660 \mathrm{~g}$ & 10. $10.1021 / 1 \mathrm{a} 201504 \mathrm{~d}$ \\
\hline 11. $10.1021 / \mathrm{nn} 202786 \mathrm{~g}$ & 12. $10.1021 / \mathrm{nn} 405236 \mathrm{k}$ \\
\hline 13. $10.1021 / \mathrm{nn} 504679 \mathrm{k}$ & 14. $10.1039 / \mathrm{C} 2 \mathrm{CE} 26516 \mathrm{H}$ \\
\hline 15. $10.1039 / \mathrm{C} 4 \mathrm{RA} 11012 \mathrm{~A}$ & 16. $10.1039 / \mathrm{C} 5 \mathrm{NR} 00028 \mathrm{~A}$ \\
\hline 17. $10.1166 /$ jnn.2011.3681 & 18. \\
\hline \multicolumn{2}{|c|}{ ZnSe Database DOIs } \\
\hline 01. $10.1007 / \mathrm{s} 11051-019-4485-6$ & 02. 10.1016/j.cap.2009.07.019 \\
\hline 03. 10.1016/j.cej.2012.09.049 & 04. 10.1016/j.matchemphys.2003.11.002 \\
\hline 05. $10.1016 /$ j.mssp.2017.03.005 & 06. $10.1021 / \mathrm{cm} 050948 \mathrm{y}$ \\
\hline 07. $10.1021 / \mathrm{cm} 400799 \mathrm{e}$ & 08. 10.1021/jp1044282 \\
\hline 09. $10.1021 / \mathrm{jp} 906812 \mathrm{t}$ & 10. $10.1039 / \mathrm{B} 917674 \mathrm{H}$ \\
\hline 11. $10.1039 / \mathrm{C} 0 \mathrm{DT} 00709 \mathrm{~A}$ & 12. $10.1088 / 0022-3727 / 42 / 12 / 125410$ \\
\hline 13. $10.5012 /$ bkcs.2005.26.11.1776 & 14. \\
\hline
\end{tabular}

